\title{
New product arrivals for spring
}

The newest laboratory products for spring include reagents for making probes for cellular oncogenes, a chamber for oxygen consumption experiments and a device for cutting Parafilm.

THE BladeRunner from Kontes makes Parafilm cutting easier (Reader Service No. 100). With the BladeRunner, Parafilm can be cut in squares or strips, with one hand. The BladeRunner's floating blade prevents the cut edge of the Parafilm from sticking to the backing, so uniform, straight cuts can be made at any length. The cutting head automatically returns after a cut for convenient repetitive dispensing, and the blades can be replaced with standard single-edge razor blades. The BladeRunner accommodates both two- and four-inch rolls of Parafilm, and costs $\$ 165$ (US).

Millipore has announced the availability of its MilliBlot systems for nucleic acid and protein transfers (Reader Service No. 101). The MilliBlot systems are designed for use with Millipore's Immobilon family of transfer membranes, but

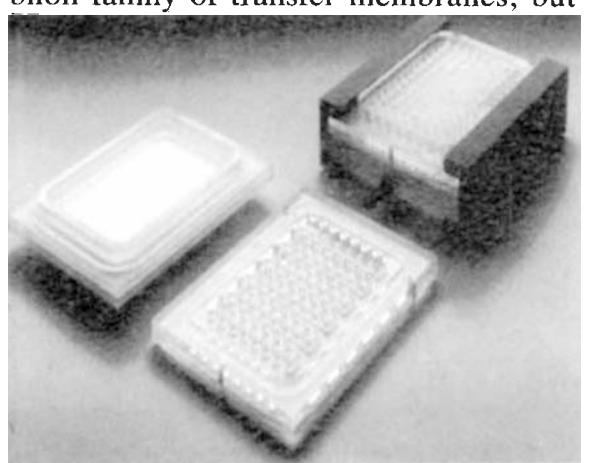

The modular MilliBlot from Millipore.

may also be used with most other commonly used transfer membranes. The $\$ 900$ (US) MilliBlot systems accommodate gels and membranes of up to $10.5 \mathrm{~cm}$ $\times 7 \mathrm{~cm}$. The modular systems allow nucleic acid transfer, 96-well dot blotting, and 48-well slot blotting with a simple change of module. Millipore says transfers can be completed in as little as 30 minutes, and no power source or lengthy overnight transfers are required.

Sigma has a specialized catalogue on cell culture reagents that's just off the presses, to accompany its comprehensive annual catalogue (Reader Service No. 102). The catalogue lists products ranging from powdered and liquid media, to more unique products such as Sigma's hybridoma-tested reagents and cell culturetested biochemicals. An extensive group of serum replacements, media for serumfree applications and numerous varieties of sera are described, along with items such as microcarrier beads, pipettes, soaps and detergents. The catalogue also includes a wealth of technical information and application methods.

For prolonged oxygen consumption experiments, Columbus Instruments International Corp. has a new airtight

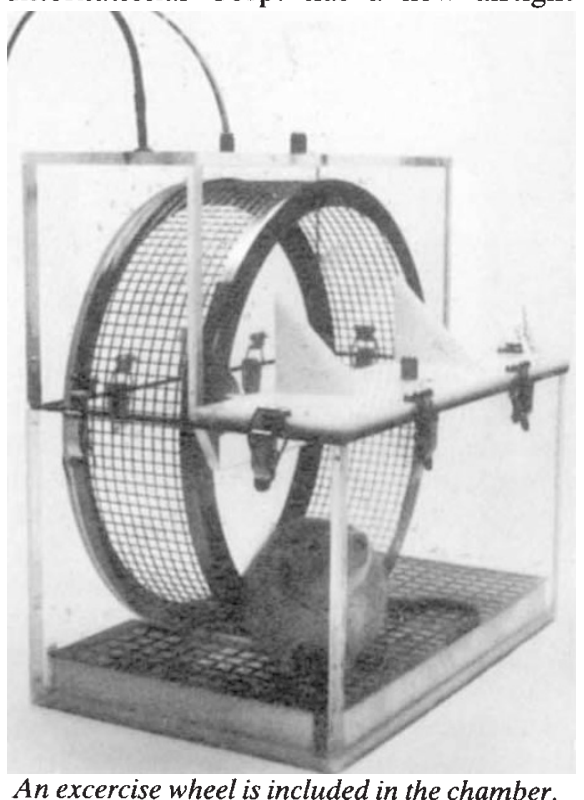

An excercise wheel is included in the chamber. acrylic chamber for small laboratory animals (Reader Service No. 103). The chamber is equipped with a voluntary exercise wheel and its size conforms to US National Institutes of Health welfare guidelines for prolonged animal housing. The exercise wheel is made of stainless steel, and has an electronic counter for keeping track of the number of rotations. One to four chambers may be connected to Columbus Instruments' Oxymax oxygen consumption computer for the collection of data on oxygen consumption, $\mathrm{CO}_{2}$ production, respiratory exchange ratio and animal ambulatory and exercise activity. The chamber's dimensions are $15.5 \times$ $9.75 \times 15.75$ inches, and each costs $\$ 1,990$ (US).

Plasmid DNAs for the preparation of RNA probes for cellular oncogenes and prostaglandin synthase are now available from Oxford Biomedical Research (Reader Service No. 104). The probes can be used for the identification and localization of human c-myc, N-myc and v-myb oncogenes and their transcription products, and for prostaglandin synthase. The plasmid DNAs direct the synthesis of RNA+ or RNA - probes using bacteriophage SP6 and T7 RNA polymerases, respectively. Biotinylated c-myc, N-myc and $\mathrm{v}-m y b \mathrm{RNA}+$ and RNA - probes have also been developed. Oxford Biomedical sells the plasmid DNAs for $\$ 95$ for $5 \mu \mathrm{g}$ and $\$ 180$ (US) for $10 \mu \mathrm{g}$. The company plans to offer several more plasmid DNAs for cellular oncogenes in the near future.

\section{Setting it straight}

The photograph published with a review of the synthetic peptide service offered by Applied Biosystems (Nature 331, 462; 1988) was printed in error. The photo depicts the OPC cartridges sold by the company.

These notes are compiled by Carol Ezzell from information provided by the manufacturers. To obtain further details about these products, use the reader service card bound inside the journal. Prices quoted are sometimes nominal and apply only within the country indicated.

\section{ADVERTISEMENTS \\ LLPOSOMES \\ REPRODUCIBLE - HOMOGENEOUS EXTREMELY RAPID

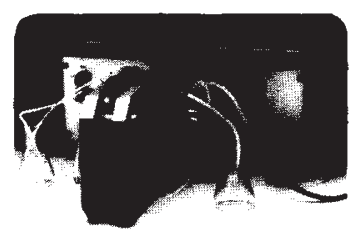

Our new LIPOSOMAT guarantees rapid $(5 \mathrm{ml}$ within 60 minutes) preparation of uniformly sized unilamellar liposomes. Liposome size is selectable between 25 and ca. $600 \mathrm{~nm}$ diameter. Sample volume is normally $5-10 \mathrm{ml}$ or up to $200 \mathrm{ml}$ if used in combination with an additional instrument. Lipid concentration can be up to $300 \mathrm{mg} / \mathrm{ml}$.

\section{GIANDENA}

POB 126, D-8000 Munich 65, FRG

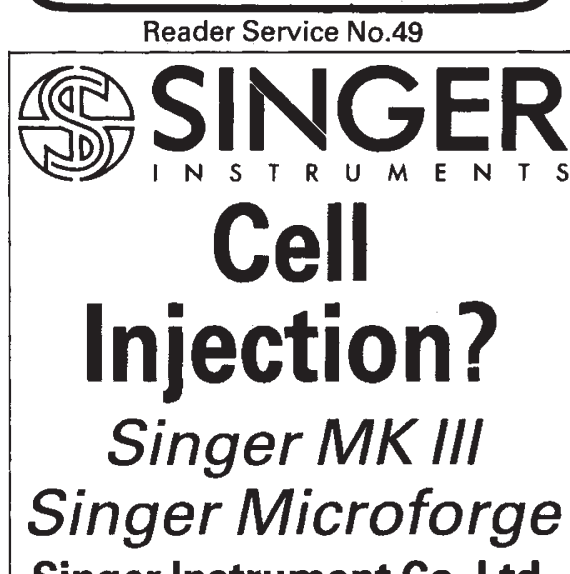

Singer Instrument Co. Ltd.

Treborough Lodge, Roadwater, Watchet,

Somerset, England, TA23 OQL.

Tel: Washford (0984) 40226

Telex: 337492 Comcab G 